\title{
Efficient interrogation method of forward Brillouin scattering in optical fibers using a narrow bandwidth long-period grating
}

\author{
L. A. SÁNCHEZ ${ }^{1}$, A. DíEz ${ }^{1,2, *}$, J. L. CRUZ ${ }^{1,2}$,AND M. V. ANDRÉs ${ }^{1,2}$ \\ ${ }^{1}$ Laboratory of Fiber Optics, ICMUV, Universidad de Valencia, Dr. Moliner 50, 46100, Burjassot, Spain \\ ${ }^{2}$ Departamento de Física Aplicada y Electromagnetismo, Universidad de Valencia, Dr. Moliner 50, 46100, Burjassot, Spain \\ *Corresponding author: antonio.diez@uv.es
}

Received XX Month XXXX; revisedXX Month, XXXX; accepted XX MonthXXXX; posted XX Month XXXX (Doc. ID XXXXX); published XX Month XXXX

\begin{abstract}
A new technique for the characterization of the effective refractive index modulation in optical fibers due to transverse acoustic mode resonances excited by electrostriction is reported. Resonances excited by an optical pulse are probed by a narrow bandwidth longperiod grating (LPG) inscribed in the fiber, which is interrogated by a CW beam. The LPG used in this experiment has a narrow bandwidth and high sensitivity to small mode index perturbations, allowing the measurement of index variations from below $10^{-9}$ to $10^{-6}$. Radial and torsional-radial acoustic modes were characterized up to $1.1 \mathrm{GHz}$. The linewidth of resonances was found to be much shorter than in previous reports in which long fiber lengths are typically required, obtaining $\mathbf{Q}$ factors as high as 5000.
\end{abstract}

http://dx.doi.org/10.1364/OL.99.099999

Forward scattering of a light wave by transverse acoustic waves inside optical fibers is a phenomenon that has been studied since the mid-80s [1]. This effect is known as forward stimulated Brillouin scattering (FSBS) or guided acoustic-wave Brillouin scattering (GAWBS). In standard optical fibers it generates frequency shifts in the range between $30 \mathrm{MHz}$ and $1100 \mathrm{MHz}$. FSBS has also been observed in microfibers [2] and in different types of microstructured optical fibers [3-5].

Transverse acoustic waves in optical fibers are excited by electrostriction when an optical pulse (the pump) propagates in the fiber [6-10]. The vibrational modes behind this interaction are the radial modes $R_{o m}$ and the torsional-radial modes $T R_{2 m}$ [11]. $R_{o m}$ are also known as polarized GAWBS modes and their displacement vector only contains the radial component depending only on the radial coordinate. $T R_{2 m}$ are known as depolarized GAWBS modes and present radial and angular components depending on both radial and angular coordinates [1].
Electrostrictively-excited $T R_{2 m}$ modes are polarization-dependent and can be suppressed using proper polarization for the pump wave [12]. Polarized and depolarized GAWBS modes are dependent on temperature and tensile strain applied to the fiber and they can be used as sensors [13-15]. Damping of the acoustic wave depends on the reflectance of the outer cladding boundary so acoustic impedance of the surrounding medium can be derived from the temporal response [16,17]. Recently, distributed sensing based on FSBS has been demonstrated $[18,19]$.

These acoustic waves can be described by density fluctuations in the optical fiber which lead to perturbations in the effective index of the optical mode through the photo-elastic effect. Quantitative knowledge of the effective index perturbation can be useful and will allow predicting the impact of this nonlinear effect. The scattering efficiencies associated to this type of interaction are small, in the order of $10^{-10} \mathrm{~m}^{-1}[1,20]$. $T R_{2 m}$ modes are easier to be observed since their interaction also produces modulation of the polarization state of the scattered light [20]. However, the detection of the modulation induced by radial modes is not straightforward and typically it requires interferometric techniques and hundreds of meters of fiber [21,22]. Unjacketing the fiber reduces the damping of the acoustic waves, and $R_{0 m}$ modes can be observed with few tens of meters of fiber-[16,23]. All these detection techniques are mostly based on the direct analysis of the light scattered by the acoustic waves. Here we propose an alternative method employing a long-period fiber grating (LPG), which is used to translate effective index modulation into amplitude modulation of a probe beam. We demonstrate the detection of both $R_{0 m}$ and $T R_{2 m}$ acoustic modes excited optically by electrostriction.

LPGs are fiber devices that couple energy from the fundamental optical core mode to co-propagating cladding modes. LPGs are based on a periodic modulation of the core refractive index that enables this coupling. The transmission spectrum presents several attenuation notches corresponding to the excitation of different cladding modes. The wavelength of resonance is given by $\lambda_{R}=\delta n_{e f f} \Lambda$, where $\delta n_{e f f}$ is the difference between the effective 
index of coupled core and cladding modes, and $\Lambda$ is the period of the grating. LPGs are frequently used as sensors due to their high sensitivity to the fiber properties and/or to external medium changes [24,25]. Chemical and biological sensors have been developed based on LPGs. High sensitivity and narrow bandwidth LPGs are required for this type of applications, as discussed in [26].

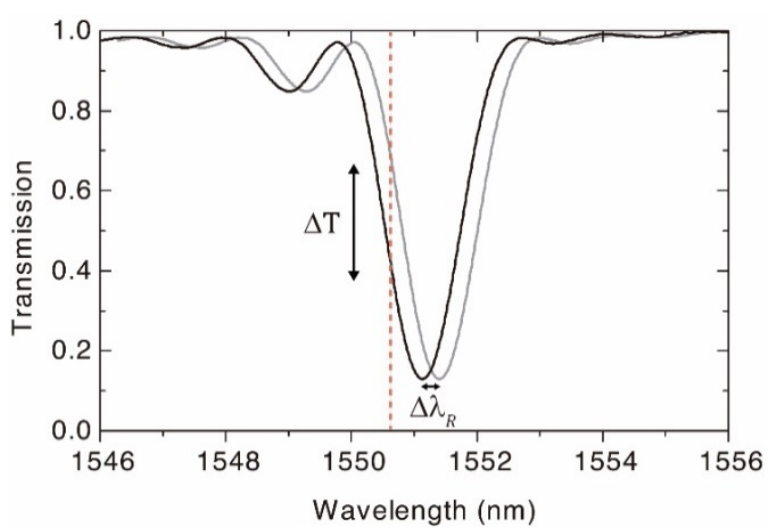

Fig. 1. Operation principle of the pump and probe technique. The vertical dashed line indicates the probe laser wavelength which is set in the linear region of the LPG. The acoustic wave displaces the resonance wavelength of the grating and the transmission at the probe wavelength changes.

The acoustic pulse generated by electrostriction at the core of the fiber by an optical pump pulse, modifies the refractive index profile of the whole fiber section. However, the strongest effect is observed when the acoustic pulse is located at the core of the fiber. Such refractive index perturbation affects essentially the effective index of the core mode since the sensitivity of cladding modes to changes of core refractive index is few orders of magnitude smaller. The operation principle is illustrated in Fig. 1. The perturbation in the effective index of the core optical mode as a result of the acoustic wave causes the shift of the notch, thus modifying the transmission of a probe laser. If the probe laser wavelength is located at one of the linear regions of the LPG, then the change in transmission is proportional to the displacement of the resonant wavelength. If the variation of the effective index of the cladding mode is neglected, the core effective index change $\Delta n_{c o}$ and the change in transmittance $\Delta T$ are given by:

$$
\Delta n_{c o}=-\Delta T /(s \cdot \Lambda)
$$

where $s$ is the slope of the notch edges in the linear region (negative at the left-side and positive at the right-side). The detection limit of the technique, i.e. the smallest value of $\Delta n_{c o}$ that can be detected, depends strongly on the spectral characteristics of the grating. The narrower is the LPG notch, the higher is $s$, and then the better is the detection limit. In this experimental proposal, the performance of the LPG is crucial, because the refractive index perturbations induced by the acoustic pulse are certainly small. In this regard, the use of fibers with large numerical aperture (NA) is preferred [27]. Alternatively, acoustic gratings and fiber Bragg gratings could substitute the LPG, enabling the characterization of low NA single mode fibers using the technique presented here.

The experimental setup is shown in Fig. 2. A Q-switched Nd:YAG microchip laser (TEEM Photonics SNP-20F-100) was used as the pump beam to generate the acoustic waves in the fiber where the
LPG was written. It emits linearly polarized pulses at $1064 \mathrm{~nm}$ with pulse duration of $700 \mathrm{ps}$ and repetition rate of $19 \mathrm{kHz}$. The pump laser was coupled to the grating using a $\times 16$ aspheric lens. A half-wave plate at the output of the pump laser allowed rotating the polarization plane of the pump. The transmission of the LPG was interrogated using a CW tunable laser (Keysight 81940A) that emits from $1520 \mathrm{~nm}$ to $1630 \mathrm{~nm}$. A polarization controller (PC) was to located at the output of the probe laser to match the principal axes of the LPG. The two optical beams were launched simultaneously through the LPG fiber in a counter-propagating configuration. Several wavelength-dependent components were inserted to combine/split the two optical beams. The transmission response of the probe laser was measured using a fast photodetector (Thorlabs DET01CFC) and an oscilloscope (Keysight DSOS104A). All the temporal traces were averaged over 1064 pulses.

Narrow bandwidth LPGs were designed to exhibit narrowband notches with very large sensitivity to small changes of the core refractive index. They were fabricated following the methods described in [27]. The spectrum of the LPG used in this experiment is shown in Fig. 1. It was written in a high NA fiber supplied by Fibercore (product number SM1500-4.2/125, NA=0.29, cutoff wavelength $1387 \mathrm{~nm}$ ). The period of the LPG was $52.3 \mu \mathrm{m}$ with a length of $11 \mathrm{~cm}$, a $3-\mathrm{dB}$ bandwidth of $1.3 \mathrm{~nm}$ and a depth of $-9 \mathrm{~dB}$. The slope at the linear regions of the LPG notch spectrum are $s=-0.98 \mathrm{~nm}^{-1}$ for the left side, and $s=0.90 \mathrm{~nm}^{-1}$ for the right side. The polymer jacket of the fiber was removed for the inscription of the LPG, and the subsequent experiments were performed with the fiber uncoated.

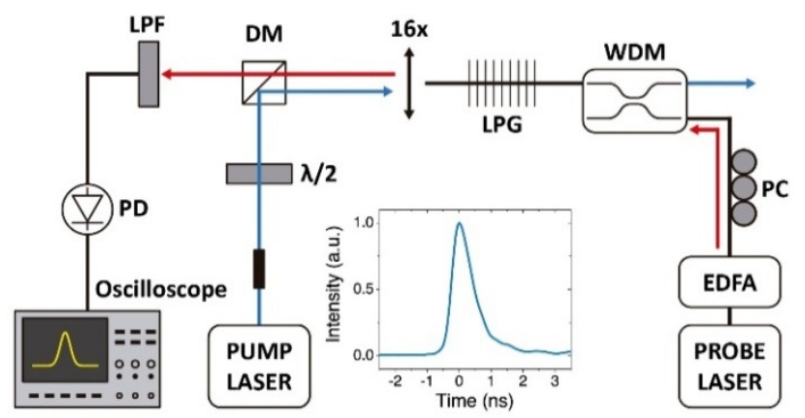

Fig. 2. Experimental setup. DM: dichroic mirror; LPF: long-pass filter; WDM: wavelength division multiplexer. Inset graph shows the temporal profile of the pump pulse.

Figure 3(a) shows a typical temporal trace of the probe laser that was recorded in the oscilloscope after a pump pulse propagated along the LPG. The probe laser wavelength was tuned to match the linear part of the left-side of the LPG notch, and the pump peak power was $7.4 \mathrm{~kW}$ The first $20 \mathrm{~ns}$ of the temporal trace were discarded since it also includes the contribution of the instantaneous response of the grating due to Kerr nonlinearity [28]. The trace shows a sequence of beats (oscillations) with amplitude decaying with time. The decay time of the envelope was $554.6 \pm 0.3$ ns. Taking into account that the period between consecutive pump pulses was $52.6 \mu$ s, the response of different pump pulses do not overlap in time. Figures 3(b) and 3(c) shows the first oscillations for two different polarization orientations of the pump, either to avoid or to favor the excitation of $T R_{2 m}$ modes 

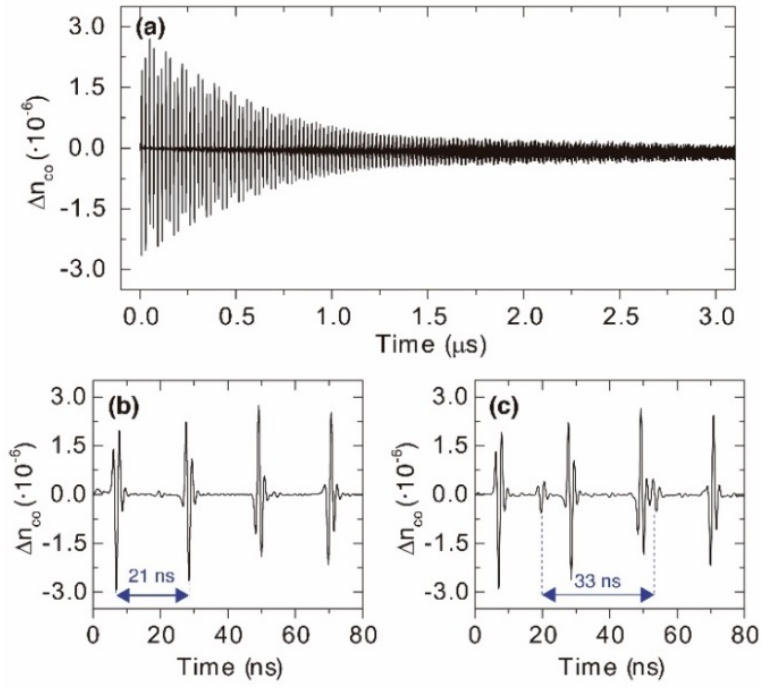

Fig. 3. (a) Core effective index variation of the LPG for a pump peak power of $7.4 \mathrm{~kW}$. (b) Detailed plot of the first $80 \mathrm{~ns}$ with negligible excitation of $T R_{2 m}$ modes and (c) with maximum excitation of $T R_{2 m}$ modes.
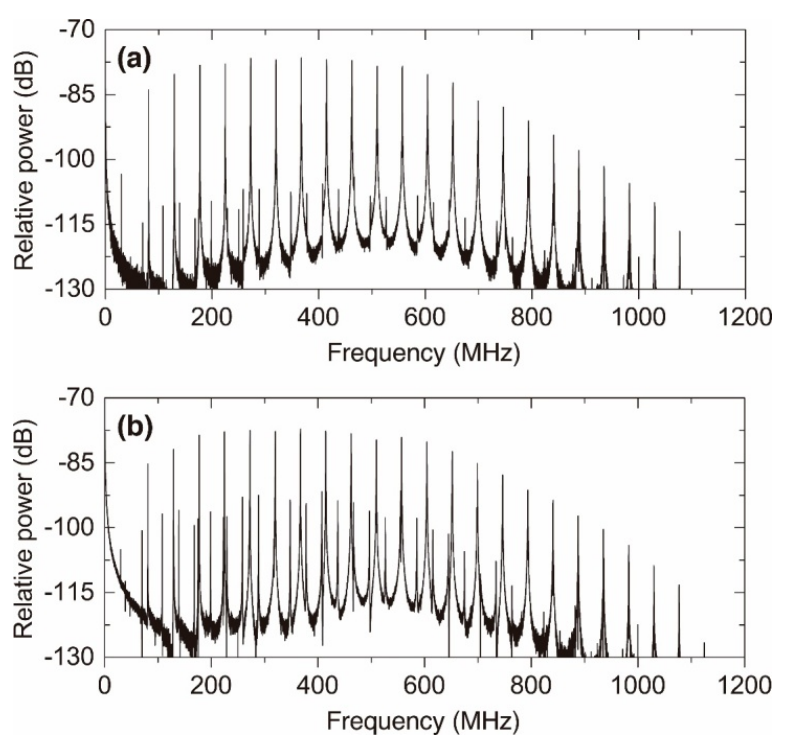

Fig. 4. Frequency response of the LPG to the acoustic wave for (a) attenuated $T R_{2 m}$ modes and (b) excited $T R_{2 m}$ modes.

respectively. Purely radial modes produce a periodic pattern of beats separated by $\sim 21 \mathrm{ns,}$ while torsional-radial modes contribute with a $\sim 33 \mathrm{~ns}$ periodic pattern. For a $125 \mu \mathrm{m}$ diameter silica optical fiber, such periods of time agree with the roundtrip time of an acoustic pulse travelling in the radial direction across the fiber at the longitudinal acoustic velocity $\left(V_{L}\right)$ and at the shear velocity $\left(V_{S}\right)$, respectively.

The RF spectra for both pump polarization orientations are plotted in Figs. 4(a) and 4(b), respectively. They were obtained performing a Fourier transform over the full temporal trace. The spectra consist on a series of peaks located at the resonance frequencies of the different acoustic mode resonances that are excited by the pump. Highest intensity peaks correspond to $R_{0 m}$ modes and are quasi-evenly spaced by $47.6 \mathrm{MHz}$. Secondary peaks located between the purely radial resonances correspond to $T R_{2 m}$ modes. Comparing both spectra an extinction ratio of $\sim 13 \mathrm{~dB}$ is obtained for the torsional-radial modes by varying the polarization orientation of the pump pulse. The presence of $T R_{2 m}$ modes can be further reduced by using circularly polarized light [12].

High-resolution spectra of individual $R_{0 m}$ modes were obtained from a signal analyzer (Keysight N9010A) with a resolution bandwidth of $15 \mathrm{kHz}$ and a video bandwidth of $100 \mathrm{~Hz}$. For this measurement, no temporal gating was applied, thus the contribution of the grating response due to Kerr nonlinearity is also included. The resonances exhibit an asymmetric Fano shape, especially for higher order modes, that we believe it is caused by the Kerr effect [22]. As an example, inset of Fig. 5 shows the spectrum of the $R_{05}$ resonance. The linewidth of the $R_{0 m}$ resonances were obtained from fitting the spectrum of each resonance to Breit-Wigner-Fano functions, yielding increasing values of full width half maximum (FWHM) from $23.9 \pm 0.1 \mathrm{kHz}\left(R_{02}\right)$ to $407.3 \pm 4.9 \mathrm{kHz}\left(R_{020}\right)$ as shown in Fig. 5. These values are considerably smaller than those previously obtained using interferometric techniques. To our knowledge, the narrowest resonances observed until now were reported in [17]; the linewidth of $R_{07}$ mode retrieved from their experiment in $15 \mathrm{~m}$ of uncoated fiber was $0.1 \mathrm{MHz}$. In our experiments the linewidth of the $R_{07}$ resonance is $82 \mathrm{kHz}$.

The linewidth of the acoustic modes resonances depends on various physical phenomena present in the fiber. The different contributions were traditionally classified as an inhomogeneous term, a viscose damping term, and a surface damping term $[5,22]$. The inhomogeneous term accounts for structural non-uniformities along the fiber length, essentially fiber diameter fluctuations. The viscose damping term is related to the lifetime of acoustic phonons in bulk silica, and the surface damping term includes the effect of the acoustic impedance mismatch at the fiber surface and the damping due to acoustic absorption of the plastic jacket in case of coated fibers. In the previously discussed experiments $[5,22]$ the main sources for the spectral broadening of the resonances were inhomogeneities in the fiber radius due to the large fiber lengths required, and also surface damping due to the fiber coating in experiments with coated fibers.

The method reported here uses a section of uncoated fiber of few $\mathrm{cm}$ in length, i.e., the LPG's length. Diameter fluctuations in such fiber length scale in conventional single-mode fibers are in the order of few $\mathrm{nm}$ [29]. The frequency shift $\Delta f$ experienced by an $R_{0 m}$ mode of resonant frequency $f$ when a small change of fiber radius $\Delta a$ is assumed, is given by $(\Delta f / f) / \Delta a \sim 1.6 \times 10^{-5} \mathrm{~nm}^{-1}$. Therefore, a deviation fiber radius $\Delta a=5 \mathrm{~nm}$, means a linewidth broadening of $6.5 \mathrm{kHz}$ and $75 \mathrm{kHz}$ for modes $R_{02}$ and $R_{020}$, respectively. The linewidth contribution due to surface damping for the uncoated fiber is given by the difference of impedances between silica and air [17]. Assuming $Z_{\mathrm{SiO}_{2}}=13.1 \times$ $10^{6} \mathrm{~kg} \mathrm{~m}^{-2} \mathrm{~s}^{-1}$ and $Z_{\text {air }}=409.4 \mathrm{~kg} \mathrm{~m}^{-2} \mathrm{~s}^{-1}$, it gives a contribution of $947 \mathrm{~Hz}$. Finally, the viscous damping term is related to the lifetime of acoustic phonons in bulk silica. In the literature, such term has been estimated in the order of a hundred $\mathrm{kHz}[5,22]$. Therefore, we can conclude that the dominant contribution to the linewidth in our results is the viscous damping term, in contrast to previous reports in which long fiber lengths are required and fiber non-uniformity is one of the main terms that contribute to the resonances broadening.

The dependence of the modes amplitude on the input pump power was studied using neutral density filters. Figure 6(a) shows 
the amplitude of the $R_{05}$ mode as a function of pump peak power. A linear dependence was found between both magnitudes in agreement with the theory $[6,10]$. The same relation was observed for modes of different orders. The linear behavior observed throughout the power range of our experiments ensured the linear response of the grating even for large index changes that occur at high pump peak powers. The smallest core effective index change measured was of the order of $10^{-9}$ corresponding to a pump peak power of $30 \mathrm{~W}$ (Figs. 6(b) and 6(c)). Reliable measurements with lower pump power could not be performed due to limitations of our experimental equipment for measuring smaller pump powers.

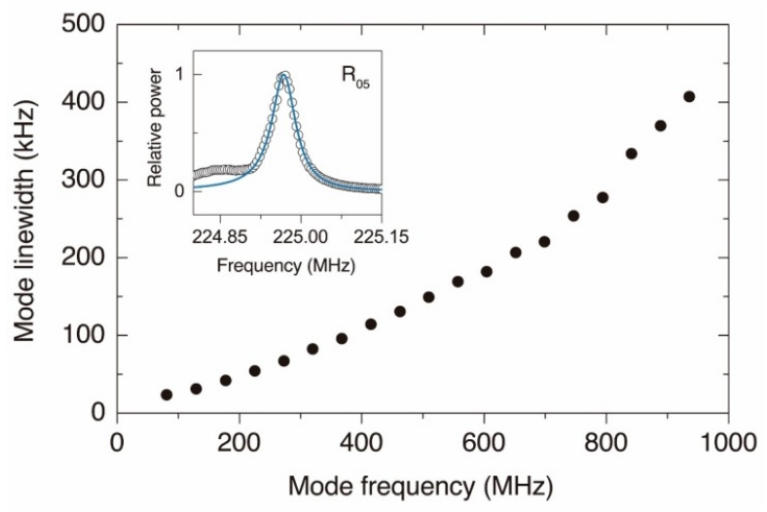

Fig. 5. Linewidth of $R_{0 m}$ modes as a function of their resonance frequency. The inset shows the $R_{05}$ spectrum and its Fano fit.
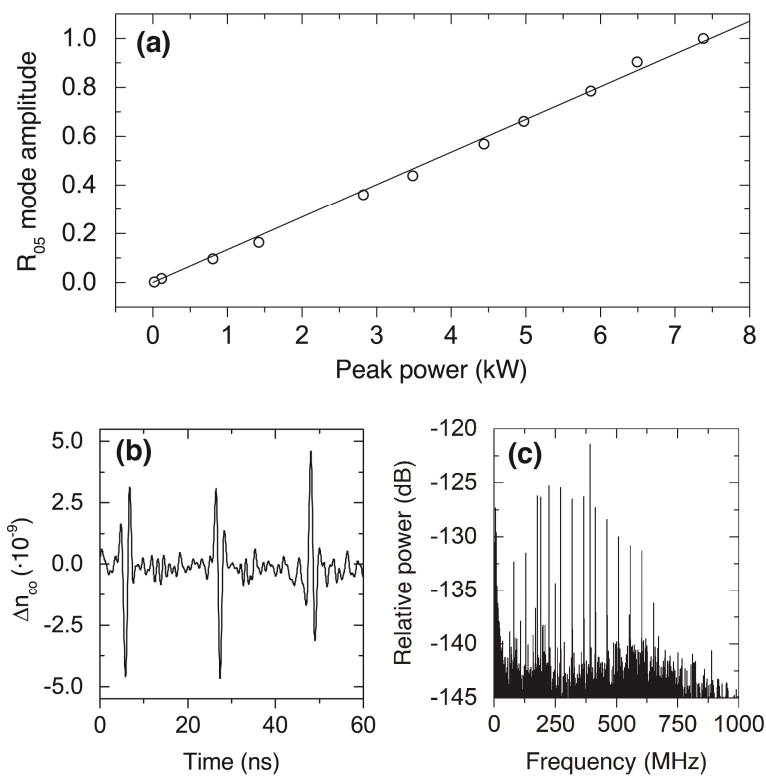

Fig. 6. (a) Normalized amplitude of $R_{05}$ mode as a function of peak power in the LPG. (b) Core effective index change and (c) the corresponding RF spectrum, for a peak power of $30 \mathrm{~W}$.

In summary, we have demonstrated a new method for the characterization of FSBS in optical fibers by means of the effective refractive index modulation generated by the transverse acoustic waves, using a narrowband LPG. We found that the linewidth of the acoustic resonances is shorter than in previous reports due to the short fiber section required by the present method that reduces significantly the structural inhomogeneities contribution to linewidth broadening .The smallest core effective index change measured was in the order of $10^{-9}$ corresponding to acoustic mode resonances excited by pump pulses of $30 \mathrm{~W}$ peak power.

Funding. Ministerio de Ciencia e Innovación- Fondo Europeo de Desarrollo Regional (PDI2019-104276RB-I00); Generalitat Valenciana (PROMETEO/2019/048).

Disclosures. The authors declare no conflicts of interest.

\section{REFERENCES}

1. R. M. Shelby, M. D. Levenson, and P. W. Bayer, Phys. Rev. B 31(8), 52445252 (1985).

2. M. S. Kang, A. Brenn, G. S. Wiederhecker, and P. S. J. Russell, Appl. Phys. Lett. 93(13), 65-68 (2008).

3. P. Dainese, P. S. J. Russell, G. S. Wiederhecker, N. Joly, H. L. Fragnito, V. Laude, and A. Khelif, Opt. Express 14(9), 4141 (2006).

4. J.-C. Beugnot, T. Sylvestre, H. Maillotte, G. Mélin, and V. Laude, Opt. Lett. 32(1), 17 (2007).

5. M. S. Kang, A. Nazarkin, A. Brenn, and P. S. J. Russell, Nat. Phys. 5(4), 276280 (2009).

6. E. M. Dianov, A. V. Luchnikov, A. N. Pilipetskii, and A. N. Starodumov, Opt. Lett. 15(6), 314 (1990).

7. E. M. Dianov, A. V. Luchnikov, A. N. Pilipetskii, and A. M. Prokhorov, Appl. Phys. B Photophysics Laser Chem. 54(2), 175-180 (1992).

8. A. Melloni, M. Frasca, A. Garavaglia, A. Tonini, and M. Martinelli, Opt. Lett. 23(9), 691 (1998).

9. Y. Jaouën, L. du Mouza, and G. Debarge, Opt. Lett. 23(15), 1185 (1998).

10. A. S. Biryukov, M. E. Sukharev, and E. M. Dianov, Quantum Electron. 32(9), 765-775 (2002).

11. R. N. Thurston, J. Sound Vib. 159(3), 441-467 (1992).

12. E. A. Golovchenko and A. N. Pilipetskii, J. Light. Technol. 12(6), 1052-1056 (1994).

13. Y. Tanaka and K. Ogusu, IEEE Photonics Technol. Lett. 10(12), 1769-1771 (1998).

14. Y. Tanaka and K. Ogusu, IEEE Photonics Technol. Lett. 11(7), 865-867 (1999).

15. N. Hayashi, K. Suzuki, S. Y. Set, and S. Yamashita, Appl. Phys. Express 10(9), (2017).

16. Y. Antman, A. Clain, Y. London, and A. Zadok, Optica 3(5), 510 (2016).

17. D. M. Chow, Z. Yang, M. A. Soto, and L. Thévenaz, Nat. Commun. 9(1), 2990 (2018).

18. G. Bashan, H. H. Diamandi, Y. London, E. Preter, A. Zadok, Nat. Commun. 9, 2991 (2018)

19. Chao Pang, Zijie Hua, Dengwang Zhou, Hongying Zhang, Liang Chen, Xiaoyi Bao, and Yongkang Dong, Optica 7, 176-184 (2020).

20. A. J. Poustie, Opt. Lett. 17(8), 574 (1992).

21. P. D. Townsend, A. J. Poustie, P. J. Hardman, and K. J. Blow, Opt. Lett. 21(5), 333 (1996).

22. J. Wang, Y. Zhu, R. Zhang, and D. J. Gauthier, Opt. Express 19(6), 5339 (2011).

23. A. J. Poustie, J. Opt. Soc. Am. B 10(4), 691 (1993).

24. V. Bhatia, Opt. Express 4(11), 457 (1999).

25. X. Shu, L. Zhang, and I. Bennion, J. Light. Technol. 20(2), 255-266 (2002).

26. M. Delgado-Pinar, Q. Shi, L. Poveda-Wong, E. Delgado-Pinar, B. Xu, J. Zhao, J. L. Cruz, and M. V. Andrés, IEEE Sens. J. 17(17), 5503-5509 (2017).

27. L. Poveda-Wong, J. L. Cruz, M. Delgado-Pinar, X. Roselló-Mechó, A. Díez, and M. V. Andrés, Opt. Lett. 42(7), 1265 (2017).

28. E. Rivera-Pérez, A. Carrascosa, A. Díez, E. P. Alcusa-Sáez, and M. V. Andrés, Appl. Phys. Lett. 113(1), 1-4 (2018).

29. E. P. Alcusa-Sáez, A. Díez, M. González-Herráez, and M. V. Andrés, Opt. Express 23(6), 7345 (2015). 


\section{List of references}

1. R. M. Shelby, M. D. Levenson, and P. W. Bayer, "Guided acousticwave Brillouin scattering," Phys. Rev. B 31(8), 5244-5252 (1985).

2. M. S. Kang, A. Brenn, G. S. Wiederhecker, and P. S. J. Russell, "Optical excitation and characterization of gigahertz acoustic resonances in optical fiber tapers," Appl. Phys. Lett. 93(13), 65-68 (2008).

3. P. Dainese, P. S. J. Russell, G. S. Wiederhecker, N. Joly, H. L. Fragnito, V. Laude, and A. Khelif, "Raman-like light scattering from acoustic phonons in photonic crystal fiber," Opt. Express 14(9), 4141 (2006).

4. J.-C. Beugnot, T. Sylvestre, H. Maillotte, G. Mélin, and V. Laude, "Guided acoustic wave Brillouin scattering in photonic crystal fibers," Opt. Lett. 32(1), 17 (2007).

5. M. S. Kang, A. Nazarkin, A. Brenn, and P. S. J. Russell, "Tightly trapped acoustic phonons in photonic crystal fibres as highly nonlinear artificial Raman oscillators," Nat. Phys. 5(4), 276-280 (2009).

6. E. M. Dianov, A. V. Luchnikov, A. N. Pilipetskii, and A. N. Starodumov, "Electrostriction mechanism of soliton interaction in optical fibers," Opt. Lett. 15(6), 314 (1990).

7. E. M. Dianov, A. V. Luchnikov, A. N. Pilipetskii, and A. M. Prokhorov, "Long-range interaction of picosecond solitons through excitation of Acoustic Waves in Optical Fibers," Appl. Phys. B Photophysics Laser Chem. 54(2), 175-180 (1992).

8. A. Melloni, M. Frasca, A. Garavaglia, A. Tonini, and M. Martinelli, "Direct measurement of electrostriction in optical fibers," Opt. Lett. 23(9), 691 (1998).

9. Y. Jaouën, L. du Mouza, and G. Debarge, "Electrostriction-induced acoustic effect in ultralong-distance soliton transmission systems," Opt. Lett. 23(15), 1185 (1998).

10.A. S. Biryukov, M. E. Sukharev, and E. M. Dianov, "Excitation of sound waves upon propagation of laser pulses in optical fibres," Quantum Electron. 32(9), 765-775 (2002).

11.R. N. Thurston, "Elastic waves in rods and clad rods," J. Sound Vib. 159(3), 441-467 (1992).

12.E. A. Golovchenko and A. N. Pilipetskii, "Acoustic effect and the polarization of adjacent bits in soliton communication lines", J. Lightwave Technol. 12(6), 1052-1056 (1994).

13.Y. Tanaka and K. Ogusu, "Temperature coefficient of sideband frequencies produced by depolarized guided acoustic-wave Brillouin scattering," IEEE Photonics Technol. Lett. 10(12), 17691771 (1998).

14.Y. Tanaka and K. Ogusu, "Tensile-strain coefficient of resonance frequency of depolarized guided acoustic-wave Brillouin scattering," IEEE Photonics Technol. Lett. 11(7), 865-867 (1999).

15. N. Hayashi, K. Suzuki, S. Y. Set, and S. Yamashita, "Temperature coefficient of sideband frequency produced by polarized guided acoustic-wave Brillouin scattering in highly nonlinear fibers," Appl. Phys. Express 10(9), (2017).

16. Y. Antman, A. Clain, Y. London, and A. Zadok, "Optomechanical sensing of liquids outside standard fibers using forward stimulated Brillouin scattering," Optica 3(5), 510 (2016).
17.D. M. Chow, Z. Yang, M. A. Soto, and L. Thévenaz, "Distributed forward Brillouin sensor based on local light phase recovery," Nat. Commun. 9(1), 2990 (2018).

18.G. Bashan, H. H. Diamandi, Y. London, E. Preter, A. Zadok, "Optomechanical time-domain reflectometry," Nat. Commun. 9, 2991 (2018).

19. Chao Pang, Zijie Hua, Dengwang Zhou, Hongying Zhang, Liang Chen, Xiaoyi Bao, and Yongkang Dong, "Opto-mechanical time-domain analysis based on coherent forward stimulated Brillouin scattering probing," Optica 7, 176-184 (2020).

20.A. J. Poustie, "Guided acoustic-wave Brillouin scattering with optical pulses," Opt. Lett. 17(8), 574 (1992).

21.P. D. Townsend, A. J. Poustie, P. J. Hardman, and K. J. Blow, "Measurement of the refractive-index modulation generated by electrostriction-induced acoustic waves in optical fibers," Opt. Lett. 21(5), 333 (1996).

22.J. Wang, Y. Zhu, R. Zhang, and D. J. Gauthier, "FSBS resonances observed in a standard highly nonlinear fiber," Opt. Express 19(6), 5339 (2011).

23. A. J. Poustie, "Bandwidth and mode intensities of guided acousticwave Brillouin scattering in optical fibers," J. Opt. Soc. Am. B 10(4), 691 (1993).

24.V. Bhatia, "Applications of long-period gratings to single an multiparameter sensing," Opt. Express 4(11), 457 (1999).

25.X. Shu, L. Zhang, and I. Bennion, "Sensitivity characteristics of longperiod fiber gratings," J. Lightwave Technol. 20(2), 255-266 (2002).

26. M. Delgado-Pinar, Q. Shi, L. Poveda-Wong, E. Delgado-Pinar, B. Xu, J. Zhao, J. L. Cruz, and M. V. Andrés, "Oligonucleotide-hybridization fiber-optic biosensor using a narrow bandwidth long period grating," IEEE Sens. J. 17(17), 5503-5509 (2017).

27.L. Poveda-Wong, J. L. Cruz, M. Delgado-Pinar, X. Roselló-Mechó, A. Díez, and M. V. Andrés, "Fabrication of long period fiber gratings of subnanometric bandwidth," Opt. Lett. 42(7), 1265 (2017).

28. E. Rivera-Pérez, A. Carrascosa, A. Díez, E. P. Alcusa-Sáez, and M. V. Andrés, "An approach to the measurement of the nonlinear refractive index of very short lengths of optical fibers," Appl. Phys. Lett. 113(1), 1-4 (2018).

29. E. P. Alcusa-Sáez, A. Díez, M. González-Herráez, and M. V. Andrés, "Improved time-resolved acousto-optic technique for optical fiber analysis of axial nonuniformities by using edge interrogation," Opt. Express 23(6), 7345 (2015). 